\title{
MJN THE PERCEIVED CULTURAL BARRIERS TO EFFECTIVE COMMUNICATION TOWARDS PATIENT AMONG NON-SAUDI REGISTERED NURSES OF A PUBLIC HOSPITAL, THE KINGDOM OF SAUDI ARABIA
}

\author{
Yee Bit-Lian*1, Rosnida Abu Bakar², Shahedan Saeidin² \\ International Medical University, Malaysia \\ ${ }^{2}$ Open University Malaysia, Malaysia \\ *Corresponding Author's Email: bitlianyee@imu.edu.my
}

\begin{abstract}
Background: In the Kingdom of Saudi Arabia (KSA) health care setting effective communication is a central to the provision of compassionate and high-quality nursing care. The aim of the survey is to study the perceived cultural barriers to effective communication towards patient and knowledge towards culture working environment among non-Saudi registered nurses in a hospital in KSA. Methodology: This is crosssectional survey conducted from January 2017 to Jun 2018 thorough self-administration questionnaire. The questionnaire was distributed to 150 nurses that randomly selected from medical and surgical fields and received feedback with total number 135 nurses. Results: Most of the respondents were female 125 (92.6\%) between 26 to 30 years. In terms of years of experience, $49(36.3 \%)$ had five to ten years of experience, and the rest 89 (65.9\%) had no previous working experience in Saudi. The findings displayed that physical and mental tiredness for $55(40.7 \%)$. The second ranked respondents strongly agreed that cultural communication barriers come from hard nursing tasks 45 (33.3\%), heavy nursing workload $46(34.1 \%)$ and followed by lack of information and skills in communication for which 50 (37.0\%). Conclusion: The respondents considering communication barriers are related to personal and social characteristics, job specification, clinical situation of patients and environmental factors. There are significant results regarding the knowledge towards culture working environment in terms of nurses' perception, cultural norms and culture on life activities.
\end{abstract}

Keywords: Cultural Barriers, Communication, Non-Saudi Nurses, Saudi Arabia

\section{INTRODUCTION}

Therapeutic communication in nursing is an interactive, dynamic operation in which the nurse influences or assists patients intentionally to achieve a better understanding of the treatment process through non-verbal or verbal communication (Potter et al., Perry, Stockert \& Hall, 2014). In Saudi Arabian health care setting, expatriate nurses formed the majority of the nursing workforce in Saudi health care facilities (Almalki, FitzGerald \& Clark, 2011). These nurses bring their own sociocultural traits, beliefs, rituals, customs and behaviours, which can differ greatly from those of their local patients (Almutairi, McCarthy \& Gardner, 2015). Misunderstandings and disputes with clients due to lack of cultural skills can jeopardise patient care and could prove fatal for patients (Felemban, O’Connor \& McKenna, 2014).

Cultural, religious and spiritual beliefs can affect a patient's perception of illness and how that patient approaches treatment (Taylor, Nicolle \& Maguire, 2013). Almutairi et al. (2015) stated that KSA culture blends the Islamic faith with Saudi tribal traditions and customs, shaping and directing Saudi attitudes and behaviours. Moreover, Almutairi et al. (2015) affirmed that cultural and linguistic barriers between patients and nurses have the potential to reduce nurses' abilities to practice competently and safely. Family is very significant in Saudi Arabia. Indeed, the family remains at the core of Saudi society (Bowen, 2014). For this reason, cultural and social life in Saudi Arabia rests 
within the confines of the family and the tribe.

\section{Problem Statement}

Effective communication between nurses and patient within hospitalization is increasingly important especially when delivering nursing care and carrying out routine treatment in daily practices. It is further suggested that Saudi patients' perspectives on cultural obstacles in communicating with foreign nurses be explored. Moreover, by exploring the barriers to effective communication between non-Saudi nurses and Saudi patients, it will help in guiding the best practices through nursing education, cultural awareness, policies or guidelines in providing effective communication. This increasing trend of foreign professional nurses into the KSA health-care system has serious implications such as cultural, language and communication barriers which in turn has the potential to compromise the ability to ensure quality of care to Saudi patients (Albougami, 2015). Hence, in order to improve effective communication, researcher feels that it is necessary to study and explore the cultural barrier that hinders effective communication in multicultural nursing workforce in KSA.

\section{Literature Review}

Cultural diversity affects standards of care. Studies conducted by Almutairi et al. (2015) and Taylor et al. (2013) explore the impact of cultural diversity on standards of patient care. Alosaimi, Dyson \& Anthony (2013) explore the experiences of non-Muslim nurses ( $\mathrm{n}=$ 7) caring for Muslim patients. The findings revealed that cultural diversity and linguistic barriers have a negative effect on expatriate nurses' patient care experiences. A study by Karout et al. (2013) determined that clients faced cultural communication difficulties in some cases. These difficulties could lead to misunderstandings that could then result in diminished care provided by the nurses. Norouzinia et al., (2015), Aghabarari, Shiri, Karimi and Samami (2015) reported that native patients were less accepting of nurses from different cultures; this reduced effective communication and affected the services delivered to patients negatively.

From a patient safety aspect, poor nurse-patient communication leads both directly and indirectly to patient harm (Lee, Allen \& Daly, 2012). When nurses understand their patients' cultural backgrounds, they can provide better care and support, avoiding misunderstandings with patients and their families. Barriers to communication among patients and nurses can cause avoidable errors, such as inadequate care, discomfort, pain and even death (Almutairi, 2015).
Possible barriers to active nurse-patient communication in health care environments can involve traditions and cultural beliefs, gender, language and accent, and spiritual and religious background. Vicencio (2014) reported that nurses lacked of knowledge about the various Muslim beliefs and spiritual practices.

Clear communication creates rapport and earns a patient's trust. In Saudi Arabia, expatriate nurses' languages and accents differ from those of the native Saudi patients, impairing these nurses' abilities to communicate efficiently with their clients. McCarthy, Cassidy, Graham McCarthy et al., Tuohy (2013) reported that the nurses in the study declared they encountered difficulties in communicating with patients who did not speak the same language. Language barriers caused anxieties about nurses' capability to conduct the assessment that forms the basis of quality health care. Religious and spiritual beliefs are important in the lives of many Saudi patients; and any deficiencies in knowledge about patients' beliefs can undermine patient care. Plaza del Pino, Soriano \& Higginbottom (2013) identified several hurdles inhibiting successful communication among nurses and their clients, including customs and religious beliefs.

Studies have noted that linguistic and cultural variations remain the biggest obstacles to effective communication. According to Nies \& McEwen (2014), nurses must interact sensitively, effectively and professionally with patients from diverse cultural, racial and ethnic backgrounds. Cultural competency is the ability to work productively within the cultural contexts of patients from different cultural backgrounds and, further, to appreciate those differences (Maurer \& Smith, 2014). Effective communication in nursing practice requires excellent communication skills, including the proper attitude and knowledge to care for patients of different cultural backgrounds (Almutairi et al., 2015).

\section{METHODOLOGY}

\section{Design}

This is a descriptive, cross-sectional quantitative study to examine the cultural interaction between expatriate nurses and Saudi patients. Data was collected during January 2017 to Jun 2018.

\section{Setting}

A public tertiary health care centre in KSA was chosen because majority of nursing staff members are expatriates, who are mostly originating from the Malaysia, Philippines, Somalia, South Africa and Europe. 


\section{Instrument}

\section{Nurse Self-Administered Communication Survey (NSACS)}

This study adopted the Nurse Self-Administered Communication Survey (NSACS) developed by Anoosheh et al. (2009).

Table 1: The NSACS's five-point Likert-type scale is constructed as follows

\begin{tabular}{|c|c|}
\hline Score & Justification \\
\hline 1 & Strongly disagree \\
\hline 2 & Somewhat Disagree \\
\hline 3 & Neither agree nor disagree \\
\hline 4 & Somewhat Agree \\
\hline 5 & Strongly Agree \\
\hline
\end{tabular}

\section{Transcultural Self - Efficacy Tool (TSET)}

The TSET was designed on the basis of relevant available educational literature that drew on four main content areas: transcultural nursing, self-efficacy theory, cultural issues in nursing care and instrumentation and includes 83 items presented in three subscales sequentially (Jeffreys \& Smodlaka, 1996, 1998). The survey instrument was divided into three sections: nurses' perceptions (11 questions), impact of culture on life activities (9 questions) and clients' cultural norms (11 questions).

\section{Validity and Reliability}

The NSACS survey's reliability was 0.96 using Cronbach's alpha coefficient (Anoosheh et al., 2009). Cronbach alpha $(\alpha)$ values were checked prior to using Elsheikh's modified version of the Affective subscale in this current study. The three subscales of the modified TSET affective survey exhibited reliability with a Cronbach $\alpha$ coefficient of 0.84 for the nurses' perceptions subscale, 0.89 for the impact of culture on life activities subscales and 0.89 for the clients' cultural norms subscale.

\section{Ethical Consideration}

All the information given by respondents was kept as confidential and the researcher would maintain anonymity of the participants involved. The respondents' names would not be recorded; respondents were instructed not to write their names on the surveys, to protect their privacy and confidentiality.

\section{RESULTS}

Data analysis was done by using SPSS Version 22. One hundred and thirty-five $(\mathrm{n}=135)$ completed survey instruments were received.

\section{Sociodemographic Characteristic of Respondents}

Majority were female nurses ( $\mathrm{n}=125,92.6 \%), 135$ (100\%) were Asian ethnicity and Muslim by faith. Malaysian formed the majority with 83 (61.5\%) of the study population. The study showed $61(45.2 \%)$ of the respondents were in age group of $26-30$ years. In relation to the education level, diploma qualification formed the majority $78(57.8 \%)$ of the study population. Those respondents who had less than one-year experience formed $25(18.5 \%)$ of the study population, while those with 5-10 years experiences formed the majority of $49(52.6 \%)$. In term of position or nursing grade, majority of the respondents was Staff Nurse I 93 (68.9\%). With regard to previous work experience in Saudi, the study showed a large number of the respondents 89 (65.9\%) had no previous work experience in Saudi Arabia. This result indicates that some of the nurses had already been exposed to the Saudi culture before joining this hospital.

In term of continuous education received by the respondents, $130(96.3 \%)$ had attended communication skills training, $37(27.4 \%)$ attended patient safety programme and $34(25.2 \%)$ attended Saudi Arabia culture training. Unfortunately, only $8(5.99 \%)$ of the respondents received training on Arabic language course and vocabulary. While $6(4.4 \%)$ received some forms of other training or courses. Thus, by and large most of the respondents had been given communication skills training.

Table 2: Socio-Demographic Data of Respondents $(n=135)$

\begin{tabular}{|l|c|c|c|}
\hline Socio-Demographic Data & & Frequency & Percent \\
\hline Gender & Male & 10 & 7.4 \\
\cline { 2 - 4 } & Female & 125 & 92.6 \\
\hline Religion & Islam & 135 & 100.0 \\
\hline Ethnic & Asian & 135 & 100.0 \\
\hline \multirow{4}{*}{ Origin } & Philippines & 52 & 38.5 \\
\cline { 2 - 4 } & Malaysia & 83 & 61.5 \\
\hline \multirow{4}{*}{ Age } & 26 - 30 Years & 61 & 45.2 \\
\cline { 2 - 4 } & $31-35$ Years & 37 & 27.4 \\
\cline { 2 - 4 } & $36-40$ Years & 33 & 24.4 \\
\cline { 2 - 4 } & $>41$ years & 4 & 3.0 \\
\hline \multirow{5}{*}{ Education } & Diploma & 78 & 57.8 \\
\cline { 2 - 4 } & Degree & 42 & 31.1 \\
\cline { 2 - 4 } & Master & 15 & 11.1 \\
\hline
\end{tabular}




\begin{tabular}{|l|c|c|c|}
\hline \multirow{2}{*}{$\begin{array}{l}\text { Years of Nusing Experience } \\
\text { in Saudi }\end{array}$} & $<1$ year & 25 & 18.5 \\
\cline { 2 - 4 } & 1-5 years & 46 & 34.1 \\
\cline { 2 - 4 } & 5-10 years & 49 & 36.3 \\
\cline { 2 - 4 } & > than 10 years & 15 & 11.1 \\
\hline Position/Nursing Grade & $\begin{array}{c}\text { Staff Nurse I } \\
\text { Staff Nurse II }\end{array}$ & 93 & 68.9 \\
& Yes & 46 & 34.0 \\
\hline $\begin{array}{l}\text { Have you worked in Saudi } \\
\text { Arabia before }\end{array}$ & 89 & 65.9 \\
\cline { 2 - 4 } & No & 130 & 96.3 \\
\hline Continuous Education Training & 37 & 27.4 \\
\hline Communication Skills & 34 & 25.2 \\
\hline Patient Safety Training/ Policies & 8 & 5.9 \\
\hline Saudi Arabia Culture Program & 6 & 4.4 \\
\hline $\begin{array}{l}\text { Arabic Language Courses / Vocabulary } \\
\text { Training }\end{array}$ & & \\
\hline Others & & \\
\hline
\end{tabular}

\section{Nurse's Self Administration Communication Survey (NSACS)}

\section{Job Specification}

The most frequent cultural communication barrier from the nurses 'somewhat agreed' was "nursing burn out" due to physical and mental tiredness for $55(40.7 \%)$ nurses. The second ranked respondents strongly agreed that cultural communication barriers come from hard nursing task 45 (33.3\%), heavy nursing workload 46 $(34.1 \%)$ and followed by lack of information \& skills in communication for which $50(37.0 \%)$.

Table 3: Frequency and Percentage of Job Specification (n=135)

\begin{tabular}{|c|c|c|c|c|c|c|c|c|c|c|}
\hline \multirow{3}{*}{ Survey Items } & \multicolumn{10}{|c|}{ Job Specification } \\
\hline & \multicolumn{2}{|c|}{ Strongly Disagree } & \multicolumn{2}{|c|}{$\begin{array}{c}\text { Somewhat } \\
\text { Disagree }\end{array}$} & \multicolumn{2}{|c|}{$\begin{array}{l}\text { NeitherAgree nor } \\
\text { Disagree }\end{array}$} & \multicolumn{2}{|c|}{ SomewhatAgree } & \multicolumn{2}{|c|}{ Strongly Agree } \\
\hline & $\mathbf{N}$ & $\%$ & $\mathbf{N}$ & $\%$ & $\mathbf{N}$ & $\%$ & $\mathbf{N}$ & $\%$ & $\mathbf{N}$ & $\%$ \\
\hline Lack of welfare facilities for nurses & 0 & 0 & 20 & 14.8 & 29 & 21.5 & 50 & 37.0 & 36 & 26.7 \\
\hline Low salary & 2 & 1.5 & 31 & 23.0 & 47 & 34.8 & 24 & 17.8 & 31 & 23.0 \\
\hline Hard nursing task & 1 & 0.7 & 15 & 11.1 & 38 & 28.1 & 36 & 26.7 & 45 & 33.3 \\
\hline Heavy nursing workload & 0 & 0 & 7 & 5.2 & 42 & 31.1 & 40 & 29.6 & 46 & 34.1 \\
\hline Nursing shift work & 2 & 1.5 & 17 & 12.6 & 56 & 41.5 & 22 & 16.3 & 38 & 28.1 \\
\hline Lack of interest to work & 2 & 1.5 & 32 & 23.7 & 48 & 35.6 & 22 & 16.3 & 31 & 23.0 \\
\hline $\begin{array}{l}\text { Nurses burn out (physical and mental } \\
\text { tiredness) }\end{array}$ & 1 & 0.7 & 16 & 11.9 & 18 & 13.3 & 55 & 40.7 & 45 & 33.3 \\
\hline Patient contact with different nurses & 0 & 0 & 17 & 12.6 & 38 & 28.1 & 46 & 34.1 & 34 & 25.2 \\
\hline $\begin{array}{l}\text { Lack of information \& skills in } \\
\text { communication }\end{array}$ & 2 & 1.5 & 21 & 15.6 & 25 & 18.5 & 37 & 27.4 & 50 & 37.0 \\
\hline
\end{tabular}

Accordingly, regarding the statement low salary 47 (34.8\%), nursing work shift $56(41.5 \%)$ and lack of interest of work $48(35.6 \%)$, the respondents neither agree nor disagree pertaining to that statements. The results showed that the respondents strongly agreed that lack of information 50 (37\%); heavy nursing workload 46 (34.1\%); and hard nursing task $45(33.3 \%$,) were job factors contributing to ineffective communication. They also somewhat agreed nurses burn out (physical and mental tiredness) 55 (40.7\%); lack of welfare facilities for nurses $50(37 \%)$ and patient contact with different nurses $46(34.1 \%)$ were most frequently identified job factors to be the barriers to effective communication.

\section{Environmental Factors}

Table 4 shows the respondents strongly agreed that lack of continuing education in communication skills $57(42.4 \%)$; lack of educational background in communication skills 49 (36.3\%); lack of nurses participating in decision making $49(36.3 \%)$; and feeling of injustice at workplace 47 (34.8\%) were the environmental factors frequently cited to be the barriers to effective communication 
Table 4: Frequency and Percentage of Environmental Factors (n=135)

\begin{tabular}{|c|c|c|c|c|c|c|c|c|c|c|}
\hline \multirow{3}{*}{ Survey Items } & \multicolumn{10}{|c|}{ Environmental Factors } \\
\hline & \multicolumn{2}{|c|}{ Strongly Disagree } & \multicolumn{2}{|c|}{$\begin{array}{l}\text { Somewhat } \\
\text { Disagree }\end{array}$} & \multicolumn{2}{|c|}{$\begin{array}{l}\text { NeitherAgree nor } \\
\text { Disagree }\end{array}$} & \multicolumn{2}{|c|}{ SomewhatAgree } & \multicolumn{2}{|c|}{ StronglyAgree } \\
\hline & $\mathbf{N}$ & $\%$ & $\mathbf{N}$ & $\%$ & $\mathbf{N}$ & $\%$ & $\mathbf{N}$ & $\%$ & $\mathbf{N}$ & $\%$ \\
\hline $\begin{array}{l}\text { Lack of educational background in } \\
\text { communication skills }\end{array}$ & 2 & 1.5 & 6 & 4.4 & 32 & 23.7 & 46 & 34.1 & 49 & 36.3 \\
\hline $\begin{array}{l}\text { Lack of continuing education in } \\
\text { communication skills }\end{array}$ & 8 & 5.9 & 5 & 3.7 & 23 & 17.0 & 42 & 31.1 & 57 & 42.2 \\
\hline $\begin{array}{l}\text { Lack of welfare and medical fa cilities for } \\
\text { patients }\end{array}$ & 3 & 2.2 & 10 & 7.4 & 28 & 20.7 & 51 & 37.8 & 43 & 31.9 \\
\hline Poor sanitation in patients' room & 2 & 1.5 & 31 & 23.0 & 42 & 31.1 & 25 & 18.5 & 35 & 25.9 \\
\hline Feeling of injustices at workplace & 0 & 0 & 8 & 5.9 & 35 & 25.9 & 45 & 33.3 & 47 & 34.8 \\
\hline Lack of managerial appreciation from nurses & 0 & 0 & 10 & 7.4 & 49 & 36.3 & 37 & 27.4 & 39 & 28.9 \\
\hline $\begin{array}{l}\text { Lack of nurses' participation in decision } \\
\text { making }\end{array}$ & 2 & 1.5 & 11 & 8.1 & 37 & 27.4 & 36 & 26.7 & 49 & 36.3 \\
\hline
\end{tabular}

While they somewhat agreed that there is lack of welfare and medical facilities for patients $51(37.8 \%)$. However about 49 (36.3\%) respondents neither agree nor disagree lack of managerial appreciation from nurses and poor sanitation in patients' room $42(31.1 \%)$ is factor that influences effective communication between expatriate nurses and Saudi patients. However, $31(23 \%)$ somewhat disagreed with regard to poor sanitation in patients' room would affect effective communication between expatriate nurses and Saudi patients.

\section{Modified Transcultural Self-Efficacy Tools (TEST)}

Due to the words constraint, only selected results are shared in this publication.

Table 5: The Relationship between Sociodemographic Characteristic \& Job Specification (Chi Square Test)

\begin{tabular}{|c|c|c|c|c|c|c|c|}
\hline \multirow{3}{*}{ Factors } & \multirow{3}{*}{ Group } & \multicolumn{4}{|c|}{ Job Specification } & \multirow{3}{*}{$\chi^{2}$} & \multirow{3}{*}{$P$} \\
\hline & & \multicolumn{2}{|c|}{ Disagree } & \multicolumn{2}{|c|}{ Agree } & & \\
\hline & & $\mathbf{N}$ & $\%$ & $\mathbf{N}$ & $\%$ & & \\
\hline \multirow[t]{2}{*}{ Gender } & Male & 8 & 9.3 & 2 & 4.1 & 1.24 & 0.265 \\
\hline & Female & 78 & 90.7 & 47 & 95.9 & & \\
\hline Religion & Islam & 86 & 100.0 & 49 & 100.0 & & \\
\hline \multirow[t]{2}{*}{ Ethnic } & American & 23 & 27.1 & 0 & 0.0 & 16.006 & $0.001^{*}$ \\
\hline & Asian & 62 & 72.9 & 49 & 100.0 & & \\
\hline \multirow[t]{2}{*}{ From } & Philippines & 49 & 57.0 & 3 & 6.1 & 34.088 & $0.001^{*}$ \\
\hline & Malaysia & 37 & 43.0 & 46 & 93.9 & & \\
\hline \multirow[t]{5}{*}{ Age } & $26-30$ Years & 52 & 60.5 & 9 & 18.4 & 30.228 & $0.001^{*}$ \\
\hline & $31-35$ Years & 16 & 18.6 & 21 & 42.9 & & \\
\hline & $36-40$ Years & 14 & 16.3 & 19 & 38.8 & & \\
\hline & $>41$ years & 4 & 4.7 & 0 & 0.0 & & \\
\hline & Married & 35 & 40.7 & 39 & 79.6 & & \\
\hline \multirow[t]{4}{*}{ Education } & Diploma & 37 & 43.0 & 41 & 83.7 & 41.556 & $0.001^{*}$ \\
\hline & Degree & 41 & 47.7 & 1 & 2.0 & & \\
\hline & Postgraduate $\mathrm{D}$ egree & 6 & 7.0 & 7 & 14.3 & & \\
\hline & Masters & 2 & 2.3 & 0 & 0.0 & & \\
\hline
\end{tabular}




\begin{tabular}{|c|c|c|c|c|c|c|c|}
\hline $\begin{array}{l}\text { Years of Nursing Experience in } \\
\text { Saudi }\end{array}$ & $1-3$ month & 0 & 0.0 & 1 & 2.0 & 28.376 & $0.001^{*}$ \\
\hline & 3-6 month & 7 & 8.1 & 0 & 0.0 & & \\
\hline & 6-12 month & 16 & 18.6 & 1 & 2.0 & & \\
\hline & $1-2$ years & 2 & 2.3 & 8 & 16.3 & & \\
\hline & $2-5$ years & 19 & 22.1 & 17 & 34.7 & & \\
\hline & $5-10$ years & 34 & 39.5 & 15 & 30.6 & & \\
\hline & $>$ than 10 years & 8 & 9.3 & 7 & 14.3 & & \\
\hline \multirow[t]{5}{*}{$\begin{array}{c}\text { Your position or Nursing } \\
\text { Grade }\end{array}$} & Staff Nurse I & 52 & 60.5 & 41 & 83.7 & 40.421 & $0.001^{*}$ \\
\hline & Staff Nurse II & 30 & 34.9 & 1 & 2.0 & & \\
\hline & $\begin{array}{c}\text { Education Liaison } \\
\text { Nurse }\end{array}$ & 0 & 0.0 & 7 & 14.3 & & \\
\hline & $\begin{array}{c}\text { Clinical Resources } \\
\text { Nurses } \\
\end{array}$ & 1 & 1.2 & 0 & 0.0 & & \\
\hline & Others & 3 & 3.5 & 0 & 0.0 & & \\
\hline \multirow[t]{2}{*}{ Current Occupational Status } & Full time Employed & 84 & 97.7 & 49 & 100.0 & 1.157 & 0.282 \\
\hline & Others & 2 & 2.3 & 0 & 0.0 & & \\
\hline \multirow[t]{2}{*}{$\begin{array}{c}\text { Have you worked in Saudi } \\
\text { Arabia before }\end{array}$} & Yes & 37 & 43.0 & 9 & 18.4 & 8.447 & $0.004 *$ \\
\hline & No & 49 & 57.0 & 40 & 81.6 & & \\
\hline \multirow[t]{4}{*}{$\begin{array}{c}\text { Knowledge Towards } \\
\text { Multicultural Working } \\
\text { Environment } \\
\end{array}$} & Excellent & 26 & 30.2 & 21 & 42.9 & 9.151 & $0.027^{*}$ \\
\hline & Good & 31 & 36.0 & 20 & 40.8 & & \\
\hline & Average & 22 & 25.6 & 8 & 16.3 & & \\
\hline & Poor & 7 & 8.1 & 0 & 0.0 & & \\
\hline
\end{tabular}

*Significant $P<0.05$

As shown in Table 5, respondents' ethnic, nationality, age, education, years of work experience in Saudi, nursing position or grade indicate a significant relationship with their job specifications that could influence effective communication with Saudi patients $(p<0.001)$. Followed by previous work experience $(p<0.004)$ and their knowledge towards multicultural working environment $(p<0.027)$.

Table 6: The Relationship between Sociodemographic Characteristic and Environmental Factors (Chi Square Test)

\begin{tabular}{|c|c|c|c|c|c|c|c|}
\hline \multirow{3}{*}{ Factors } & \multirow{3}{*}{ Group } & \multicolumn{4}{|c|}{ Environmental Factors } & \multirow{3}{*}{$\chi^{2}$} & \multirow{3}{*}{$P$} \\
\hline & & \multicolumn{2}{|c|}{ Disagree } & \multicolumn{2}{|c|}{ Agree } & & \\
\hline & & $\mathbf{N}$ & $\%$ & $\mathbf{N}$ & $\%$ & & \\
\hline \multirow[t]{2}{*}{ Gender } & Male & 1 & 1.4 & 9 & 13.6 & 5.636 & $0.018^{*}$ \\
\hline & Female & 68 & 98.6 & 57 & 86.4 & & \\
\hline Religion & Islam & 69 & 100.0 & 66 & 100.0 & & \\
\hline \multirow[t]{2}{*}{ Ethnic } & American & 19 & 27.9 & 4 & 6.1 & 9.791 & $0.002 *$ \\
\hline & Asian & 49 & 72.1 & 62 & 93.9 & & \\
\hline \multirow[t]{2}{*}{ From } & Philippines & 28 & 40.6 & 24 & 36.4 & 0.253 & 0.615 \\
\hline & Malaysia & 41 & 59.4 & 42 & 63.6 & & \\
\hline
\end{tabular}


CULTURAL BARRIERS TO EFFECTIVE COMMUNICATION TOWARDS PATIENT AMONG REGISTERED NURSES IVI

\begin{tabular}{|c|c|c|c|c|c|c|c|}
\hline \multirow[t]{2}{*}{ Age } & $26-30$ Years & 35 & 50.7 & 26 & 39.4 & 2.054 & \multirow[t]{2}{*}{0.561} \\
\hline & $31-35$ Years & 18 & 26.1 & 19 & 28.8 & & \\
\hline & 36 - 40 Years & 14 & 20.3 & 19 & 28.8 & & \\
\hline & $>41$ years & 2 & 2.9 & 2 & 3.0 & & \\
\hline & Married & 37 & 53.6 & 37 & 56.1 & & \\
\hline \multirow[t]{4}{*}{ Education } & Diploma & 35 & 50.7 & 43 & 65.2 & 24.420 & $0.001 *$ \\
\hline & Degree & 31 & 44.9 & 11 & 16.7 & & \\
\hline & Postgraduate Degree & 1 & 1.4 & 12 & 18.2 & & \\
\hline & Masters & 2 & 2.9 & 0 & 0.0 & & \\
\hline \multirow{7}{*}{$\begin{array}{l}\text { Years of Nursing Experience } \\
\text { in Saudi }\end{array}$} & $1-3$ month & 0 & 0.0 & 1 & 1.5 & 27.357 & $0.001 *$ \\
\hline & 3-6 month & 7 & 10.1 & 0 & 0.0 & & \\
\hline & 6-12 month & 15 & 21.7 & 2 & 3.0 & & \\
\hline & $1-2$ years & 2 & 2.9 & 8 & 12.1 & & \\
\hline & $2-5$ years & 17 & 24.6 & 19 & 28.8 & & \\
\hline & $5-10$ years & 22 & 31.9 & 27 & 40.9 & & \\
\hline & $>$ than 10 years & 6 & 8.7 & 9 & 13.6 & & \\
\hline \multirow[t]{5}{*}{$\begin{array}{c}\text { Your Position or Nursing } \\
\text { Grade }\end{array}$} & Staff Nurse I & 48 & 69.6 & 45 & 68.2 & 15.570 & $0.004 *$ \\
\hline & Staff Nurse II & 17 & 24.6 & 14 & 21.2 & & \\
\hline & Education Liaison Nurse & 0 & 0.0 & 7 & 10.6 & & \\
\hline & $\begin{array}{c}\text { Clinical Resources } \\
\text { Nurses } \\
\end{array}$ & 1 & 1.4 & 0 & 0.0 & & \\
\hline & Others & 3 & 4.3 & 0 & 0.0 & & \\
\hline \multirow[t]{2}{*}{ Current Occupational Status } & Full time employed & 67 & 97.1 & 66 & 100.0 & 1.942 & 0.163 \\
\hline & Others & 2 & 2.9 & 0 & 0.0 & & \\
\hline \multirow[t]{2}{*}{$\begin{array}{l}\text { Have you worked in Saudi } \\
\text { Arabia before }\end{array}$} & Yes & 27 & 39.1 & 19 & 28.8 & 1.606 & 0.205 \\
\hline & No & 42 & 60.9 & 47 & 71.2 & & \\
\hline \multirow[t]{4}{*}{$\begin{array}{c}\text { Knowledge Towards } \\
\text { Multicultural Working } \\
\text { Environment } \\
\end{array}$} & Excellent & 14 & 20.3 & 33 & 50.0 & 24.356 & $0.001^{*}$ \\
\hline & Good & 26 & 37.7 & 25 & 37.9 & & \\
\hline & Average & 22 & 31.9 & 8 & 12.1 & & \\
\hline & Poor & 7 & 10.1 & 0 & 0.0 & & \\
\hline
\end{tabular}

*Significant $P<0.05$

A significant relationship can be observed from Table 6 , between respondents' gender $(p<0.018)$, ethnic $(p<0.002)$, education and years of working experience $(p<0.001)$, nursing position/nursing grade $(p<0.004)$ knowledge towards multicultural working $(p<0.001)$ and environment environmental factors in influencing effective communication. 
Table 7: The Relationship between Sociodemographic Characteristic Factors and Transcultural Self-Efficacy Tools (Chi Square Test)

\begin{tabular}{|c|c|c|c|c|c|c|c|}
\hline \multirow{3}{*}{ Factors } & \multirow{3}{*}{ Group } & \multicolumn{4}{|c|}{ Transcultural Self-Efficacy Tools } & \multirow{3}{*}{$\chi^{2}$} & \multirow{3}{*}{$\boldsymbol{P}$} \\
\hline & & \multicolumn{2}{|c|}{ Disagree } & \multicolumn{2}{|c|}{ Agree } & & \\
\hline & & $\mathbf{N}$ & $\%$ & $\mathbf{N}$ & $\%$ & & \\
\hline \multirow[t]{2}{*}{ Gender } & Male & 0 & 0.0 & 10 & 12.7 & 5.921 & $0.015^{*}$ \\
\hline & Female & 56 & 100.0 & 69 & 87.3 & & \\
\hline Religion & Islam & 56 & 100.0 & 79 & 100.0 & & \\
\hline \multirow[t]{2}{*}{ Ethnic } & American & 15 & 27.3 & 8 & 10.1 & 6.705 & $0.010^{*}$ \\
\hline & Asian & 40 & 72.7 & 71 & 89.9 & & \\
\hline \multirow[t]{2}{*}{ From } & Philippines & 26 & 46.4 & 26 & 32.9 & 2.528 & 0.112 \\
\hline & Malaysia & 30 & 53.6 & 53 & 67.1 & & \\
\hline \multirow[t]{5}{*}{ Age } & $26-30$ Years & 35 & 62.5 & 26 & 32.9 & 15.256 & $0.002 *$ \\
\hline & $31-35$ Years & 8 & 14.3 & 29 & 36.7 & & \\
\hline & $36-40$ Years & 13 & 23.2 & 20 & 25.3 & & \\
\hline & $>41$ years & 0 & 0.0 & 4 & 5.1 & & \\
\hline & Mar ried & 21 & 37.5 & 53 & 67.1 & & \\
\hline \multirow[t]{4}{*}{ Education } & Diploma & 38 & 67.9 & 40 & 50.6 & 17.768 & $0.001 *$ \\
\hline & Degree & 18 & 32.1 & 24 & 30.4 & & \\
\hline & Postgraduate Degree & 0 & 0.0 & 13 & 16.5 & & \\
\hline & Masters & 0 & 0.0 & 2 & 2.5 & & \\
\hline \multirow[t]{7}{*}{$\begin{array}{l}\text { Years of Nursing Experience } \\
\text { in Saudi } \\
\end{array}$} & $1-3$ month & 0 & 0.0 & 1 & 1.3 & 40.611 & $0.001 *$ \\
\hline & 3-6 month & 7 & 12.5 & 0 & 0.0 & & \\
\hline & 6-12 month & 15 & 26.8 & 2 & 2.5 & & \\
\hline & $1-2$ years & 3 & 5.4 & 7 & 8.9 & & \\
\hline & $2-5$ years & 6 & 10.7 & 30 & 38.0 & & \\
\hline & 5-10 years & 19 & 33.9 & 30 & 38.0 & & \\
\hline & $>$ than 10 years & 6 & 10.7 & 9 & 11.4 & & \\
\hline \multirow[t]{5}{*}{$\begin{array}{c}\text { Your Position or Nursing } \\
\text { Grade }\end{array}$} & Staff Nurse I & 42 & 75.0 & 51 & 64.6 & 14.835 & $0.005^{*}$ \\
\hline & Staff Nurse II & 11 & 19.6 & 20 & 25.3 & & \\
\hline & Education Liaison Nurse & 0 & 0.0 & 7 & 8.9 & & \\
\hline & Clinical Resources Nurses & 0 & 0.0 & 1 & 1.3 & & \\
\hline & Others & 3 & 5.4 & 0 & 0.0 & & \\
\hline \multirow[t]{2}{*}{ Current Occupational Status } & Full time employed & 54 & 96.4 & 79 & 100.0 & 0.940 & 0.332 \\
\hline & Others & 2 & 3.6 & 0 & 0.0 & & \\
\hline \multirow[t]{2}{*}{$\begin{array}{l}\text { Have you worked in Saudi } \\
\text { Arabia before }\end{array}$} & Yes & 20 & 35.7 & 26 & 32.9 & 0.024 & 0.877 \\
\hline & No & 36 & 64.3 & 53 & 67.1 & & \\
\hline \multirow[t]{4}{*}{$\begin{array}{c}\text { Knowledge Towards } \\
\text { Multicultural Working } \\
\text { Environment } \\
\end{array}$} & Excellent & 10 & 17.9 & 37 & 46.8 & 30.241 & $0.001^{*}$ \\
\hline & Good & 17 & 30.4 & 34 & 43.0 & & \\
\hline & Average & 23 & 41.1 & 7 & 8.9 & & \\
\hline & Poor & 6 & 10.7 & 1 & 1.3 & & \\
\hline
\end{tabular}


Table 7 displays a significant relationship between respondents' gender $(p<0.015)$, ethnic $(p<0.010)$, age $(p<0.002)$ education and years of experience working in Saudi $(p<0.001)$ nursing position/grade $(p<0.005)$, knowledge towards multicultural working environment $(p<0.001)$ and Transcultural Self-Efficacy Tools which were to determine level of respondents' understanding in transcultural working environment.

\section{DISCUSSION}

The results revealed that expatriate nurses noticed barriers to effective communication with their patients. The cause can be ascribed to the cultural discrepancies between non-Saudi registered nurses and their Saudi patients in the health care environment. Further, the findings show that cultural awareness, cultural skills, cultural knowledge and healthcare environment are important factors for cultural competence. They attributed this situation to many cultural barriers to effective communication factors pertaining to four section such as personal and social characteristics, job specifications, clinical situation of patients and environmental factors. Some of which are unfamiliarity with dialect, too much expectation with patients, heavy nursing workload, disease severity, and lack of continuing education in communication skills. This is similar with study by McCarthy (2013), communication obstacles, lack of training, and difficulties in communicating with patients who did not speak the same language will affect nurses' performance and effective communication between nurses and patients.

Despite the respondent's facing to many culture barriers, Transcultural Survey Efficacy Tools (TSET) survey revealed they were confident about providing nursing care for Saudi patients. It categorized into nurse perception, impact of cultures on life activities and clients culture norms. Some of which respondents are treating their clients with respect to their culture and they also understand the difference between a communication disability and a communication difference.

The result findings showed the influence of culture barriers to effective communication between nurses and patients based on personal and social characteristic where respondents were unsure and ambiguous about age, sex, nursing job, and too much expectation will effect on culture barriers under personal and social characteristic. This result is contradicting with study was done by Fakhr-Movahedi et al. (2011), where it was shown that nurses were tired of patient's attitudes towards receiving treatment and care from nurse of the opposite sex.

In general, communicating with diverse age groups has its own issues. Nurses can have good interactions with their clients through improved awareness of each age group (Bridges et al., 2013). A majority of the respondents somewhat agreed that the age of Saudi patient affected how they (nurses) communicated with them (patient). Similarly, Park \& Song (2005) and Jahromi \& Ramezanhi (2014) found that a generation gap presents a communication hindrance between nurses and patients.

Thirty-five percent of respondents indicated that nurses' nationality during patient-nurse communication has an effect. Research by Karout et al. (2013) showed that Saudi patients perceived foreign female nurses (especially Filipinos and Indians) as maids or housekeepers. Almutairi et al. (2015) found that Filipino nurses experienced a lack of respect from Saudi nationals. When nurses complained to patients that they did not follow care plans, the patients stated that they (the patients) were Saudi (Alosaimi et al., 2013).

The current research shows that poor communication between expatriate nurses and Saudi patients is due to the lack of a common language in multicultural settings, an environment common in tertiary health care. The results in this study are comparable with those obtained from nurses in the Hospital in Taif, Saudi Arabia (Al-Harasis, 2013).

In the present study, it is identified that having very sick patients in the ward or having patients with a contagious disease hindered communication. Some researchers have argued that deteriorating patient health limits nurse-patient interaction. A study conducted in Netherlands by De Graaff et al. (2012) found that communication and decision-making processes between nurses and immigrant patients suffering from incurable cancer were perceived to hinder interaction as well as effective care.

This study revealed that they experience a feeling of injustice in the workplace and a lack of managerial appreciation. Management's role has been cited in some recent studies, where the respondents have indicated that hospital management always side with local patients, resulting in them experiencing profound feelings of inequality and injustice and hindering nurse-patient interactions (Almutairi et al., 2015; Alosaimi et al., 2013; Jahromi \& Ramezanli, 2014). 
Respondents in the current study reported that a lack of participation in decision-making processes is a factor that influences nurse-patient communication. Minimal chances to engage in making decisions and consequent resentment and feelings of isolation have also been reported elsewhere in the literature (Scholtz et al., Nel, Poggenpoel, \& Myburgh, 2016). Poor nurse-doctor connection can result in the loss of professional dignity, along with a drop in the quality of care (Stievano et al., 2012).

The TSET affective survey results generally indicated that respondents were confident in giving care and were aware that cultural background and culturespecific health care can affect nursing care. Several studies on nurses' perceptions of the challenges and barriers to culturally competent care have been conducted outside KSA. Investigators in Singapore (Tay, Ang \& Hegney, 2012), Spain (Plaza del Pino et al., 2013) and the UAE (El-Amouri \& O'Neill, 2011) have documented language obstacles contributing to delivering culturally incompetent care, affecting patient-nurse communication negatively. Clearly, if nurses understand their patient's language, communication may be more effective and complications could be avoided.

In the TSET affective survey results of the current study respondents, $39.3 \%$ indicated they were confident about understanding the impact of culture on gender roles. This view is further supported by Vicencio et al. (2015), who stated (in a survey performed in Saudi Arabia) that expatriate nurses understand the culturally specific factors that could influence patient attitudes and how nurses implement nursing care. Thus, patients may benefit from pre-admission clinical education regarding the function of the nurse to understand that nurses will not challenge the patient's religion and its traditions.

As overall, the descriptive finding has revealed positive and significant results pertaining to relationship between sociodemographic characteristic such as age, years of experience, gender, qualification, position, knowledge towards multicultural working environment and continuous education received by the respondents between culture barrier to effective communication and knowledge towards culture working environment. The relationship between cultural barriers has been discussed based on personal and social factors, job specification, clinical situation of patients and environmental characteristic. Moreover, knowledge towards culture working environment is based on Transcultural Self-Efficacy Tools (TEST) such as nurses' perceptions, impact of cultures on life activities $\&$ clients' cultural norms.

In the relationship between sociodemographic and characteristic knowledge towards cultural working environment, the result noted the significant relationship between respondents' gender $(p<0.015)$, ethnic group $(p<0.010)$, age $(p<0.002)$ education and years of experience working in Saudi $(p<0.001)$ nursing position/grade $(p<0.005)$, knowledge towards multicultural working environment $(p<0.001)$ and Transcultural Self-Efficacy Tools (TEST) which were to determine level of respondents' understanding in transcultural working environment.

However, as an overall, the mean score for cultural barriers to effective communication by using Nurse's Self Administration Communication Survey (NSACS), such as personal and social characteristic, job specification clinical situation of patients and environment has been measured. The personal and social characteristic factors were highly perceived by respondents as the communication barriers between expatriate nurses and their Saudi patients.

\section{RECOMMENDATION}

\section{Pre-Departure Briefing}

All candidates are mandatory to attend predeparture briefing from every and each country. The agency must prepare it properly and it is also necessary to have a comprehensive departure checklist orientation for new nurses. The programme must be conducted at least one week and this is mandatory for them to attend without any exclusion.

\section{Overview of Saudi Working Environment}

This is considered to share presentation to welcome them to work in Saudi Arabia culture and diversity. Sharing photoshoot, presentation or video will help the candidates to be more focused. This programme can be included in the overview of Saudi culture of working environment with multicultural nationality. It can be PowerPoint slide presentation, video presentation or invitation of candidate to share the experience.

\section{Basic Arabic Language Programme}

Agency should provide at least the introduction of Arabic language that need to be practiced in daily working environment for every candidate. The course at least can be conducted within two or three days. Candidates will be benefitted from this course as a basic preparation and it will help them to increase their self- 
awareness and motivation.

\section{Hospital Orientation Programme}

The Human Resource (HR) persons should revise the orientation programme for new employee especially expatriate nurses to be more comprehensive so that they can be able to achieve an orientation objective. Presently, only English Test Proficiency (ETP) is mandatory for expatriate nurses to pass. The organization should hire Arabic educator to teach basic Arabic communication among expatriate nurses during the orientation programme or the probationary period. It can be permanently hospital programme to provide Arabic Class to expatriate nurses to improve their knowledge and train them how to speak Arabic language correctly.

\section{Nursing Education Programme}

Nursing Education Department (NED) is vital for nurse to extend their career development to become better for patient satisfaction and safety. NED must update the General Nursing Orientation (GNO) programme for new expatriate nurse and probationary period (at least within 3 months). The programme should include Basic Arabic Communication among Saudi patients in daily clinical practices. NED must delegate the best Arabic speakers as educators to conduct learning and teaching sessions.

This classroom sessions need to be continuing for senior expatriate nurse to get them too involved by simulation training activities, examination, quiz and roleplay. It will help the nurses to have more confidence, integrity, self-motivation and help them release stress, learn from one and another and have improved communication skills among Saudi patients in the working environment.

\section{Unit Orientation Programme \& Probationary}

Unit Manager \& Clinical Nurse Resource is a roleplay for newcomer to adapt within their working environment. Effective unit orientation programme should be implemented to expatriate nurses for better and effective communication between Saudi patients \& families. They have to make sure every unit must have Unit Assistant as Arabic translator to guide and support new staffs. Unit manager should provide Nurses \& Patient Educators to guide nurses during health education activities.

\section{Nursing Resources System (NRS)}

Nursing Resources System must create Committee Formation Order (CFO) to update any information related to educations link, e-learning, quiz, learning packages, dictionary, shareware folder and anything related to education purpose. All information related to communication must be easily referred and accessed by the expatriate nurses.

\section{CONCLUSION}

Several factors were identified and these factors contribute to the communication barrier between expatriate nurse and Saudi patient such as personnel demographic, personal and social characteristic, job specifications, clinical situation of patients and environment factors, nurse's perceptions, impact of culture on life activities and client's cultural norms. These difficulties can be a major obstacle for expatriate nurses and can lead to an insufficient exchange of information and poor-quality nursing care. Lacking of self-awareness, training, education, simulation and practicing form expatriate nurses will have effects on communication process and it can also reduce patient satisfaction and safety.

\section{REFERENCES}

Albougami, A. (2015). Role of language and communication in providing quality healthcare by expatriate nurses in Saudi Arabia. Journal of Health Specialties, 3(3), pp 166-172.

Al-Harasis, S. (2013). Impact of Language Barrier on Quality of Nursing Care at Armed Forces Hospitals, Taif, Saudi Arabia. Middle East Journal of Nursing, 7(4), pp 17-24.

Almalki, M., FitzGerald, G. \& Clark, M. (2011). The nursing profession in Saudi Arabia: An overview. International Nursing Review, 58(3), pp 304-311.

Almutairi, A.F., McCarthy, A. \& Gardner, G.E. (2015). Understanding cultural competence in a multicultural nursing workforce: Registered nurses' experience in Saudi Arabia. Journal of Transcultural Nursing, 26(1), pp 16-23. 
Alosaimi, D., Dyson, S. \& Anthony, D. (2013). A Phenomenological Study of Non-Muslim Nurses' Experiences of Caring for Muslim Patients in Saudi Arabia. International Journal of Arts \& Sciences, 6(2), pp 637-646.

Anoosheh, M., Zarkhah, S., Faghihzadeh, S. \& Vaismoradi, M (2009). Nurse-patient communication barriers in Iranian nursing. International Nursing Review, 56(2), pp 243-249.

Bowen, W. H. (2014). The History of Saudi Arabia, (2 ${ }^{\text {nd }}$ ed.), Greenwood, California.

Bridges, J., Nicholson, C., Maben, J., Pope, C., Flatley, M., Wilkinson, C. \& Tziggili, M. (2013). Capacity for care: meta-ethnography of acute care nurses' experiences of the nurse-patient relationship. Journal of Advanced Nursing, 69(4), pp 760-772.

De Graaff, F., Francke, A., Van Den Muijsenbergh, M. \& van Der Geest, S. (2012). Talking in triads: communication with Turkish and Moroccan immigrants in the palliative phase of cancer. Journal of Clinical Nursing, 21(21-22), pp 3143.

El-Amouri, S. \& O'Neill, S. (2011). Supporting cross-cultural communication and culturally competent care in the linguistically and culturally diverse hospital settings of UAE. Contemporary Nurse, 39(2), pp 240-255.

Fakhr-Movahedi, A., Salsali, M., Negharandeh, R. \& Rahnavard, Z. (2011). A qualitative content analysis of nursepatient communication in Iranian nursing. International Nursing Review, 58(2), pp 17-180.

Felemban, E., O’Connor, M. \& McKenna, L. (2014). Cultural view of Nursing in Saudi Arabia. Middle East Journal of Nursing, 8(4), pp 8-14.

Jahromi, M. \& Ramezanli, S. (2014). Evaluation of barriers contributing in the demonstration of an effective nursepatient communication in educational hospitals of Jahrom, 2014. Global Journal of Health Science, 6(6), pp 54.

Jeffreys, M.R. \& Smodlaka, I. (1998). Exploring the factorial composition of the transcultural self-efficacy tool. International Journal of Nursing Studies, 35(4), pp 217-225.

Jeffreys, M.R. \& Smodlaka, I. (1996). Steps of the Instrument Design Process: An Illustrative Approach for Nurse Educators. Nurse Educator, 21(6), pp 47-52.

Karout, N., Abdelaziz, S., Goda, M., Al-Tuwaijri, S., Almostafa, N., Ashour, R. \& Alradi, H. (2013). Cultural diversity: A qualitative study on Saudi Arabian women's experience and perception of maternal health services. Journal of Nursing Education and Practice, 3(11), pp 172-182.

Lee, P., Allen, K. \& Daly, M. (2012). A 'Communication and Patient Safety' training programme for all healthcare staff: can it make a difference? BMJ Quality \& Safety, 21(1), pp 84.

Maurer, F.A. \& Smith, C. M. (2014). Community/Public Health Nursing Practice: Health for Families and Populations ( $5^{\text {th }}$ ed.), Saunders, Missouri.

McCarthy, J., Cassidy, I., Graham, M.M. \& Tuohy, D. (2013). Conversations through barriers of language and interpretation. British Journal of Nursing, 22(6), pp 335-339.

Nies, M.A. \& McEwen, M. (2014). Community/Public Health Nursing: Promoting the Health of Populations (6 ${ }^{\text {th }}$ ed.). Saunders, Missouri.

Norouzinia, R., Aghabarari, M., Shiri, M., Karimi, M. \& Samami, E. (2015). Communication Barriers Perceived by Nurses and Patients. Global Journal of Health Science, 8(5), pp 65-74.

Park, E.-K. \& Song, M. (2005). Communication barriers perceived by older patients and nurses. International Journal of Nursing Studies, 42(2), pp 159-166.

Plaza del Pino, F.J., Soriano, E. \& Higginbottom, G.M. (2013). Sociocultural and linguistic boundaries influencing intercultural communication between nurses and Moroccan patients in southern Spain: A focused ethnography. BMC Nursing, pp 12-14. 
Potter, P.A., Perry, A.G., Stockert, P. \& Hall, A. (2014). Essentials for Nursing Practice ( $8^{\text {th }}$ ed.). Elsevier, Missouri.

Scholtz, S., Nel, E.W., Poggenpoel, M. \& Myburgh, C.P. (2016). The Culture of Nurses in a Critical Care Unit. Global Qualitative Nursing Research, 3, pp 1-11.

Stievano, A., Marinis, M.G.D., Russo, M.T., Rocco, G. \& Alvaro, R. (2012). Professional dignity in nursing in clinical and community workplaces. Nursing Ethics, 19(3), pp 341- 356.

Tay, L.H., Ang, E. \& Hegney, D. (2012). Nurses' perceptions of the barriers in effective communication with inpatient cancer adults in Singapore. Journal of Clinical Nursing, 21(17-18), pp 2647-2658.

Taylor, S.P., Nicolle, C. \& Maguire, M. (2013). Cross-cultural communication barriers in health care. Nursing Standard, 27(31), pp 35-43.

Vicencio, D.A., Alsulaimani, A.A., Ruiz, F.B. \& Elsheikh, H.A. (2015). Affective competency of Filipino nurses working in the hospitals of Taif City Kingdom of Saudi Arabia. International Journal of Nursing and Midwifery, $7(4), \mathrm{pp} 46-53$. 